\title{
Arthroscopic Treatment of Neer Type 2 Distal Third Clavicle Fractures with the ZipTight System
}

\section{Pablo Cañete San Pastor*}

Hospital de Manises, Valencia, Spain

*Corresponding Author: Pablo Cañete San Pastor, Hospital de Manises, Valencia, Spain.

Received: August 13, 2019; Published: August 28, 2019

\section{Abstract}

Purpose: Neer type II fractures of the external third of the clavicle are fractures with a high rate of nonunion or malunion (up to $30 \%$ ). Multiple surgical techniques have been describe their treatment. Also, several arthroscopic techniques.

Methods: We have retrospectively reviewed 29 cases with Neer type II distal third clavicle fractures treated with coracoclavicular fixation with a suspension system.

Results: All fractures except 2 have undergone complete consolidation in 3.5 months, without the disadvantages of other systems such as KW, screws or plates. The rate of consolidation has been $96.5 \%$ (all but 1 patient).The mobility obtained was very satisfactory: flexion of $176^{\circ}$, abduction of $145^{\circ}$, an external rotation of $80^{\circ}$, an internal rotation with the hand until T11 and horizontal adduction of $132^{\circ}$.

The functional results obtained have been excellent in most patients: obtaining 96 points on the Constant scale and excellent UCLA in 26 cases.

This is one of the studies with more patients treated with this type of technique. All patients have been followed until the clinical and radiographic consolidation of the fractures. We also contribute some technical modifications to the initial technique that we have been obtaining with the experience to improve the clinical and radiological result of the patients.

Conclusion: The arthroscopic assisted treatment of Neer type II distal third clavicle fractures with a Ziptight system is a reproducible technique that allows us to reduce and stabilize the fracture and also to diagnose and treat possible glenohumeral o rotator cuff injuries, obtaining excellent clinical.

Keywords: ZipTight; Neer Type II; Fractures

\section{Introduction}

Between $35 \%$ and $45 \%$ of shoulder girdle fractures are clavicle fractures, and of these, $15-25 \%$ are fractures of the distal third. Neer classified the fractures of the external third of the clavicle into three types: Type I, lateral to the coracoclavicular ligaments; Type II, medial to the coracoclavicular ligaments; and Type III, fractures affecting the acromioclavicular joint. Type II fractures are subclassified in IIA and IIB: in IIA the coracoclavicular ligaments remain inserted in the lateral fragment, and in IIB the conoid ligament (the most medial of the coracoclavicular ligaments) is broken and the trapezoid ligament (the more Lateral) remains inserted in the lateral fragment. In type II fractures, especially type IIB, the arm weight moves the lateral fragment towards distal and the trapezius moves the medial fragment in a proximal direction. This fact explains the separation of both fragments and that these are fractures with a high rate of nonunion or malunion (up to 30\%) [1-4].

Multiple surgical techniques have been describe for the treatment of these fractures: percutaneous fixation with kirschner wires, coracoclavicular screws, cerclages, open reduction and internal fixation with subacromial hook plates or precontoured 
plates of lateral third of the clavicle, reconstruction of the coracoclavicular ligaments extirpating or not the lateral fragment [5-11]. Also, several arthroscopic techniques have been described for the treatment of these fractures [12-15].

In this study we have retrospectively reviewed 29 cases with Neer type II distal third clavicle fractures treated with coracoclavicular fixation with a suspension system. All fractures except 3 have undergone complete consolidation in 3.5 months, without the disadvantages of other systems such as KW, screws or plates.

\section{Materials and Methods}

We reviewed 29 patients with displaced fractures of the distal third of clavicle, type IIB in the Neer classification, treated with arthroscopic coracoclavicular fixation with the Ziptight system (Biomet) and followed until the clinical and radiographic consolidation of the fracture. We have retrospectively studied the radiographs, the patients satisfaction, the functional result with the Constant test and the complications appeared.

\section{Technique (Video 1)}

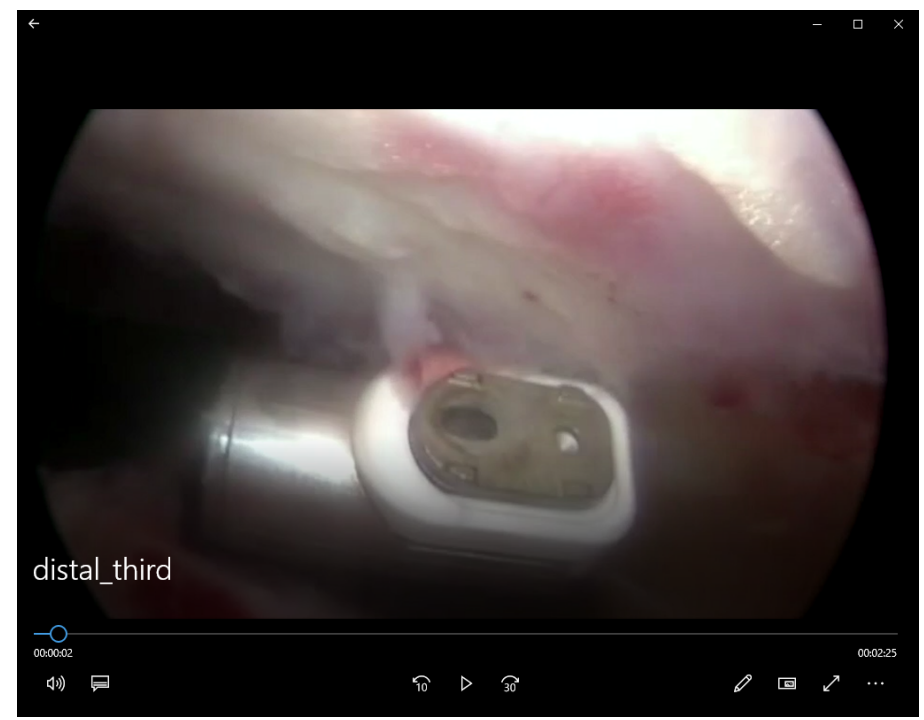

All patients underwent general and regional anesthesia. We operated the patients in a beach chair position. In order to work more confortable and to reach easier to the subcoracoid space, we apply axial traction to the arm, either with 5 kilograms of percutaneous traction or with an arm holder (Trimano) that allows us to apply axial traction and keep it in that position or remove it in the last part of the surgery, when we reduce and fix the fracture.

We create an standard posterior portal and examine the glenohumeral joint to evaluate the presence of possible associated injuries and treat them (labrum, cuff, chondral lesions). Then we access to the subacromial space and locate the coracoacromial liga- ment that will be our guide to locate the coracoid. With the scope in the posterior portal, we created a lateral portal, just on the anterior edge of the acromion. We locate the coracoacromial ligament, which we will follow from the anterior edge of the acromion to the coracoid. Once the coracoid is located, we change the scope to the lateral portal and create an anterior portal. We will clean the base of the coracoids well, to have a good view.

With the scope in the lateral portal, we insert a modified anterior cruciate ligament reconstruction guide to get the desired angle of approach (Figure 1). We will support the guide in the lower part of the base of the coracoid, centered in the mediolateral plane, to avoid fractures when passing the drill.

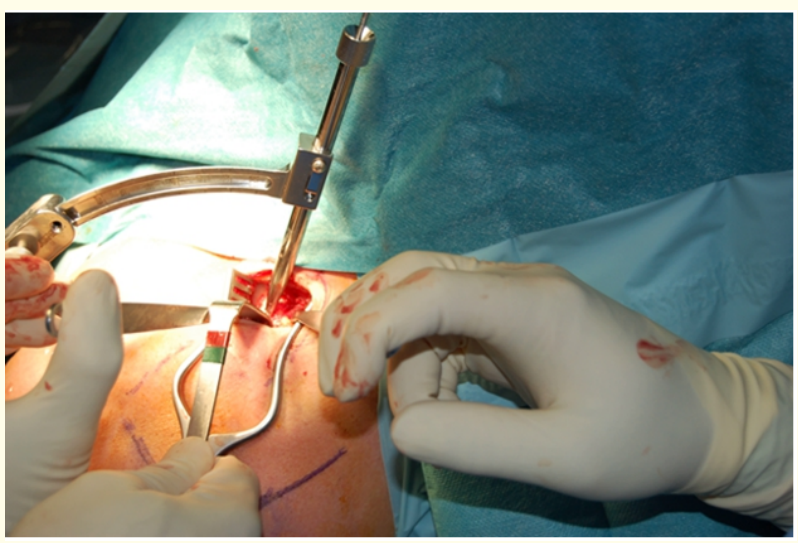

Figure 1: Modified anterior cruciate ligament guide leaning on the clavicle.

Then me make an incision of about $3 \mathrm{~cm}$, following the Lingers lines , to expose the fracture of the distal third of the clavicle.

With the scope in the lateral portal, we verified the correct placement of the foot of the guide in the base of the coracoid, and we supported the drilla guide in the diaphysis of the clavicle, 1.5 $\mathrm{cm}$ medial to the fracture. At this moment we release the traction of the arm, to facilitate the reduction of the fracture. The same guide has a locking system that facilitates reduction of the fracture in the super-inferior plane. With manual palpation and direct visualization we verify that there is no displacement of the fracture in the anteroposterior plane. We can tilt the guide to get a posteroanterior entrance from superior to inferior, moving the medial fragment to lower and anterior direction (correcting the habitual directions of the displacement of these fractures).

We introduce a pin through the guide, which will pass the two corticals of the clavicle and the two cortices of the coracoid. We 
check the correct position, centered on clavicle and coracoid, of the pin. Through the pin, we use a cannulated drill bit of $4.5 \mathrm{~mm}$ (Figure 2), to pass the 4 corticals again. We remove the pin and through the cannulated drill, we pass a suture from the clavicle to the coracoid and retrieve it through the anterior portal (Figure 3). We use this suture to pass the implant from the clavicle to the coracoid. We check with arthroscopic view the correct placement of the metallic button at the base of the coracoid, and place the other button on the sutures of the clavicular part of the implant (Figure 4). The ziptight is a self-locking implant, with the ziploop system: when pulling from the sliding sutures, reduces the rest of loops and get a reduction of the clavicle to the coracoid, thus reducing the fracture in the superoanterior plane (and also in the anteroposterior plane if we have slightly inclined the guide when making the holes). We can help the reduction by compressing the medial fragment in the anteroinferior direction and pushing the arm upwards to reduce

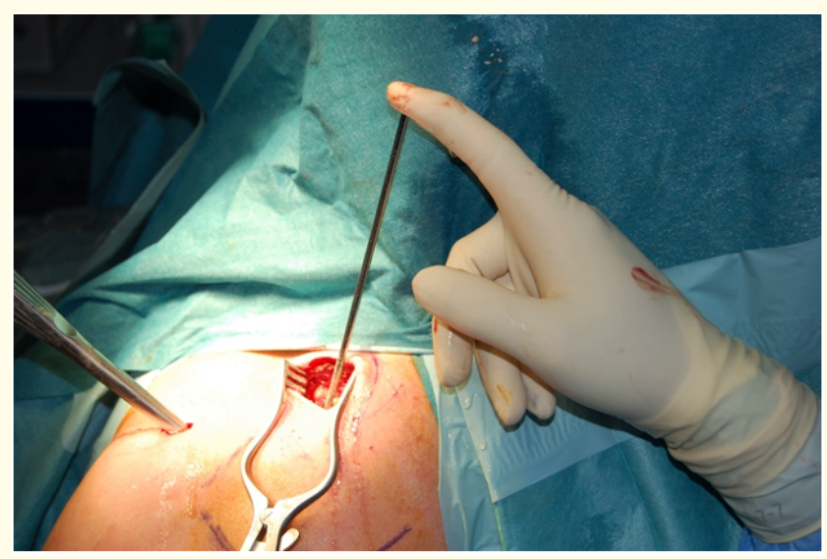

Figure 2: 4,5 canulated drill passing the two corticals of the clavicle and the two of the coracoid.

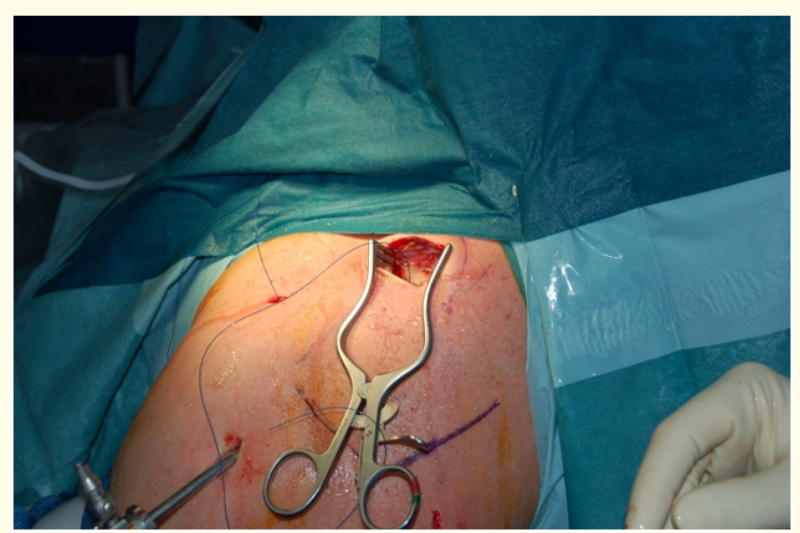

Figure 3: Shuttle suture from the clavicle to the coracoid retrieved through the anterior portal. Scope in the lateral portal.

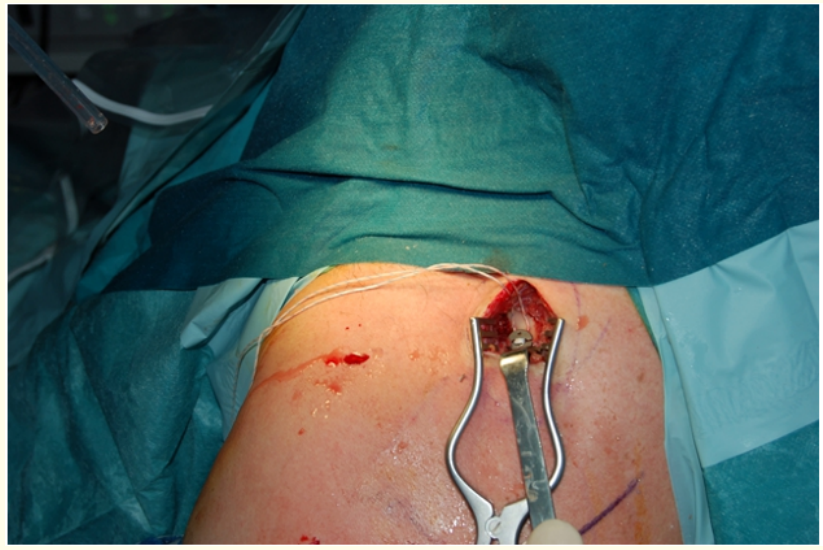

Figure 4: Button supported on the clavicle and blocking the sutures, mantaining the reduction of the fracture.

the distal fragment. The system is self-locking and requires no knots, which could cause subsequent discomfort.
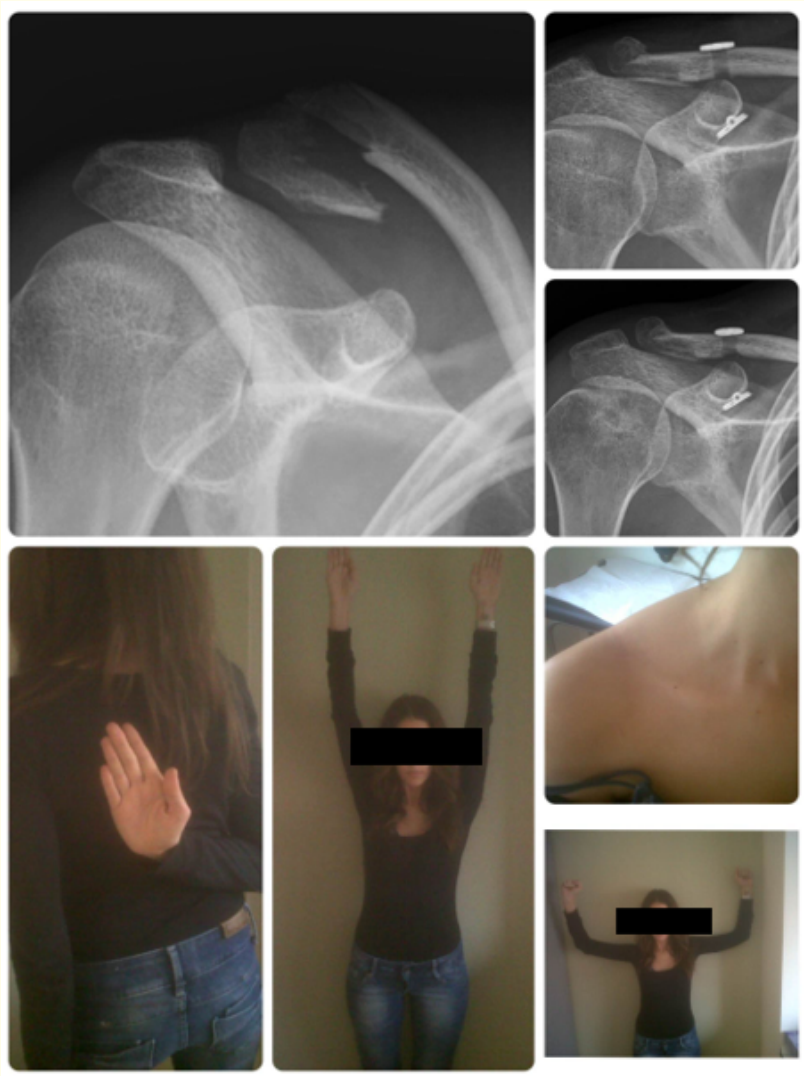

Figure 5: 1. Xray acute fracture; 2 . Inmediate postop $x$ ray control; 3.3 months $x$ ray control with the fracture healed; $4,5,6$ and 7 . Clinical control at 3 moths showing the excellent functional and aesthetic result. 
We check the reduction clinically and radiologically. Correct closure of the deltotrapezoid fascia is very important (Figure 5).

We placed a standard sling on the patient for 6 weeks. The sling can be removed for personal hygiene. Passive and self-assisted exercises begin at 3 weeks and active exercises at 6 weeks. We do not allow carry weight, do efforts or sports activities until the consolidation of the fracture is verified (about 3 months).

At this point, we add a technical note: after having three cases of delayed union due to lack of anteroposterior reduction of the fracture, (the fractures finally healed at 9,12 and 12 months (Figure 6)), we decided to add a system of reduction and fixation of the fracture in the anteroposterior plane; we did one unique case with two compression screws with bad result: the fracture didn't heal and required another surgery for plating and adding bone graft (Figure 7). Now we add an interfragmentary cerclage with high resistance suture, making one or two loops, as has been described by other authors (Figure 8). Adding this cerclage, we obtain greater
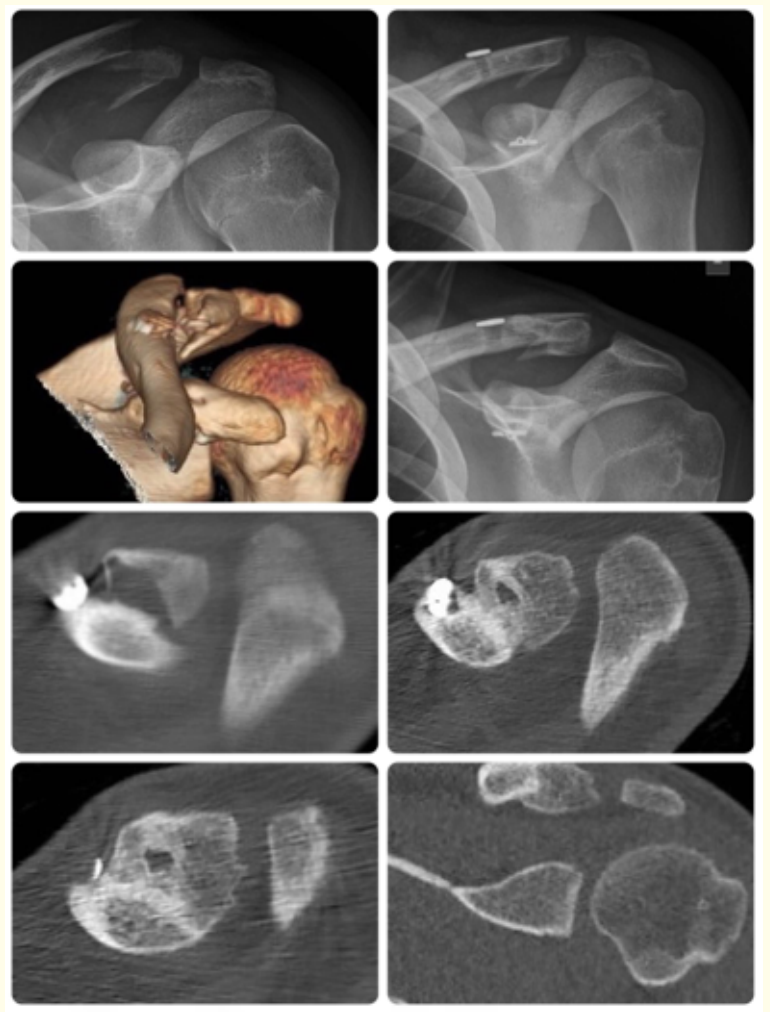

Figure 6: 1 . X ray acute fracture; 2 . Inmediate postop x ray control; 3. CT Scan after 6 months; 4 . X ray after 6 months; 5 .

CT Scan after 6 months; 6,7 and 8. CT Scan after 12 months.
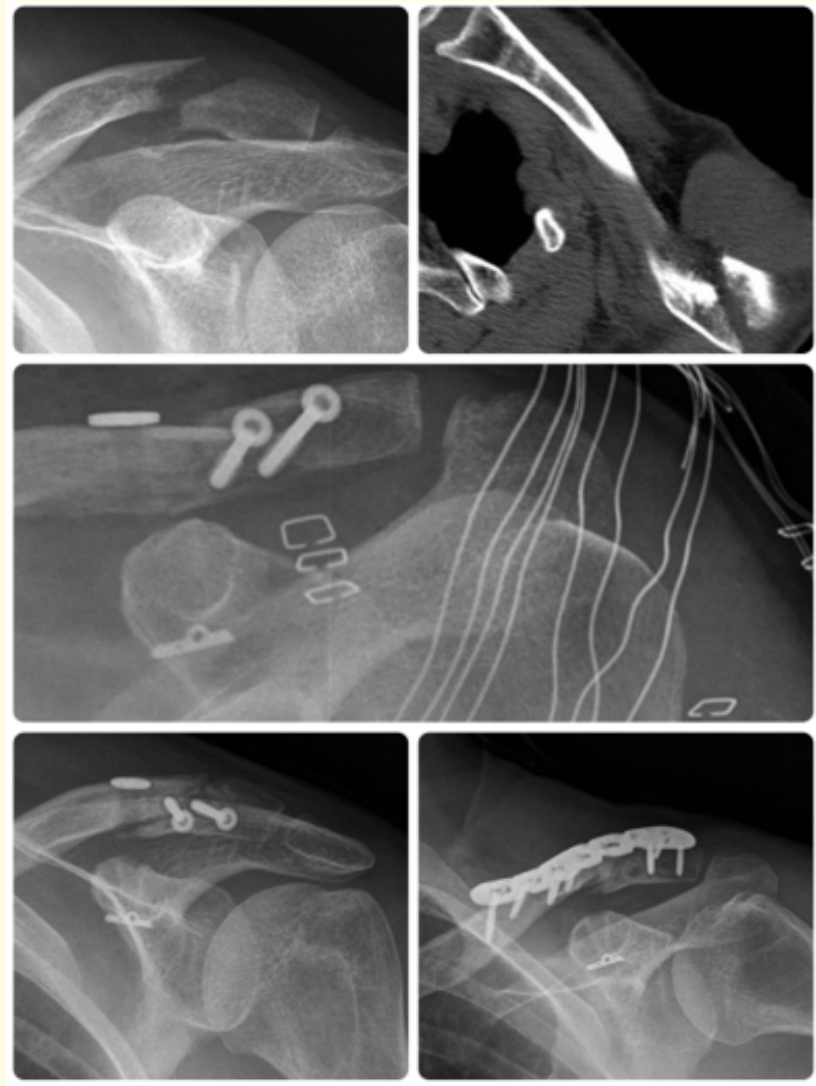

Figure 7: 1. Xray acute fracture; 2.CT Scan acute fracture with the displacement in the anteroposterior direction; 3. Inmediate postop $\mathrm{x}$ ray control with the ziptight and two anteroposterior compresion screws; 4 . Xray control after 10 months with non-union; 5. Final result with plate and bone graft, keeping the ziptight.

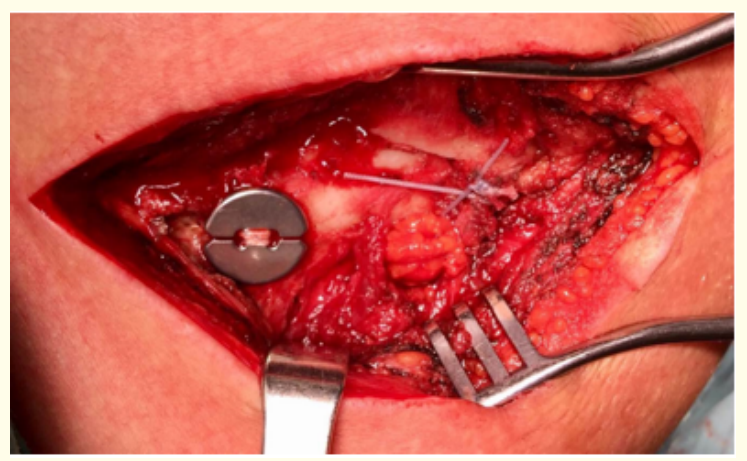

Figure 8: Image of the button on the clavicle and the interfragmentary cerclage with high streng sutures to reduce the fracture in the anteroposterior direction. 
stability and a sooner healing of the fracture. All these cases with technical variations (screw and suture cerclage) are not included in the study.

\section{Results}

We reviewed 29 patients, 21 men and 8 women. The mean age was 35.7 years (18-59). Radiographic controls were performed in the immediate postoperative period, at 2-3 weeks, 6-8 weeks, at 3 months and until consolidation. The rate of consolidation has been 96.5\% (all but 1 patient).

The average mobility obtained was very satisfactory with an anterior flexion of $176^{\circ}$, an abduction of $145^{\circ}$, an external rotation of $80^{\circ}$, an internal rotation with the hand until T11 and an horizontal adduction of $132^{\circ}$.

The functional results obtained have been excellent in most patients. We evaluated the final result of patients with the Constant and UCLA scales. Obtaining 96 points on the Constant scale and excellent UCLA in 26 cases, with full recovery at 3 months.

All patients except three achieved radiographic consolidation of the fracture at 3 months. Two patients presented a delay of consolidation, getting the heal of the fracture at 9 and 12 months, as we verified in a CT Scan (Figure 6). The third presented a non-union and required surgery to put bone graft and osteosynthesis with plate and screws.

There have been complications in two cases

- One case of low grade infection that was resolved after removal of the device at 6 months, after fracture consolidation.

- Another patient with poor outcome despite correct fracture consolidation and complete recovery of mobility. He required a second intervention for removing the device, resection of the distal third of the clavicle and anterior capsular plication because of microinstability. He later developed a scapular dyskinesia. It's the only working case in the series.

\section{Discussion}

Neer type II fractures of the distal clavicle, especially type IIB are usually displaced and unstable fractures and a non-surgical treatment causes high rates of poor functional results.

A lot of surgical techniques have been described for the treatment of these fractures, including arthroscopic assisted fixation
$[5-11]$.

The ZipTight system (reference 904834 Biomet) was designed for the stabilization of acromioclavicular dislocations. We have used this system to treat this type of fracture with excellent results, achieving a complete functional recovery of the shoulder. Studies have already been published with the use of this type of implants for distal third clavicle fractures with excellent results [12-15].

Arthroscopy is a minimally invasive technique that respects the tissues and allows us to access to the base of the coracoid with a minimum dissection, also allows us to perform a diagnosis and treatment of glenohumeral joint or rotator cuff pathology. (Burkhart reported $40 \%$ of associated injuries in acromioclavicular dislocations) [22].

The Ziptight is a fast and secure system that helps to reduce the fracture and maintain the reduction until the fracture heals. This is a self locking device that does not require knots, avoiding the inconveniences of supraclavicular knots with high resistance suture (irritation and discomfort).

Radioscopy control in the operating room could be advisable at least in the first cases, although according to experience, we do not consider it fundamental.

The coracoclavicular system reduces the fracture in the superior inferior but not in the anteroposterior plane. So its very important to control the displacement of the fracture in anteroposterior direction to avoid delays or even absences of consolidation.

We can tilt the guide to get a posteroanterior entrance from superior to inferior, while pushing the medial fragment to an inferior and anterior. With this maneuver, we achieve a better reduction and fixation of the fracture in both planes; but even with this maneuver we had two cases of delayed union and one of non-union. We decided to add a system to improve the anteroposterior reduction and the stability of the fixation; we added in 1 case (non included in the study) two anteroposterior interfragmentary screws (Figure 7), with but result. And now, we currently add a 8-sided cerclage with high strength suture between the two fragments of the clavicle (a single band or better doublé), which in addition to anteroposterior stability, provides interfragmentary compression (Figure 8) (these cases are not included in this study. We will review our cases with the new technique in order to compare the results). Several studies have reported good clinical and radiological results with Coracocla- 
vicular fixation plus fractures fixation with suture tension bands 13,18-2. Adding this cerclage, we obtain greater stability and a sooner healing of the fracture.

This technique gives us an excellent functional result, with a stable reduction avoiding the inconveniences of other systems (needle migration, plate intolerance, need to remove hook plates, osteolysis of coracoclavicular screws, loss of reduction of needles or plates). In addition to providing a good aesthetic result.

Although there are several published studies of the arthroscopic treatment of distal third clavicle fractures, this is one of the studies with more patients treated with this type of technique. All patients have been followed until the clinical and radiographic consolidation of the fractures. We also contribute some technical modifications to the initial technique that we have been obtaining with the experience to improve the clinical and radiological result of the patients.

In conclusion, the arthroscopic assisted treatment of Neer type II distal third clavicle fractures with a Ziptight system is a reproducible technique that allows us to reduce and stabilize the fracture and also to diagnose and treat possible glenohumeral o rotator cuff injuries, obtaining excellent clinical and radiological

\section{Bibliography}

1. Neer CS. "Nonunion of the clavicle". JAMA 172 (1960): 10061011.

2. Neer CS II. "Fractures of the clavicle. In: Rockwood CA Jr, Green DP, eds. Fractures in Adults". Ed 2. Philadelphia: JB Lippincott (1984): 707-713.

3. Neer CS II. "Fractures of the distal third of the clavicle". Clinical Orthopaedics and Related Research 58 (1968): 43-50.

4. Neer CS II. "Fracture of the distal clavicle with detachment of the coraco-clavicular ligaments in adults". Journal of Trauma 3 (1963): 99-110.

5. Moore T0. "Internal pin fixation for fracture of the clavicle". The American Surgeon 17 (1951): 580-583.

6. Mullaji AB and Jupiter JB. "Low-contact dynamic compression plating of the clavicle". Injury 25 (1994): 41-45.
7. Edwards DJ., et al. "Fractures of the distal clavicle: A case for fixation". Injury 23 (1992): 44-46.

8. Van der Meijden OA., et al. "Treatment of clavicle fractures: Current concepts review". Journal of Shoulder and Elbow Surgery 21 (2012): 423-429.

9. Kona J., et al. "Type II distal clavicle fractures: A retrospective review of surgical treatment". Journal of Orthopaedic Trauma 4 (1990): 115-120.

10. Levy 0. "Simple, minimally invasive surgical technique for treatment of type 2 fractures of the distal clavicle". Journal of Shoulder and Elbow Surgery 12 (2003): 24-28.

11. Ballmer FT and Gerber C. "Coracoclavicular screw fixation for unstable fractures of the distal clavicle. A report of five cases". The Journal of bone and joint surgery 73 (1991): 291294.

12. Geoffroy Nourissat MD., et al. "Arthroscopic Stabilization of Neer Type 2 Fracture of the Distal Part of the Clavicle". Artrhoscopy 23 (2007): 674.

13. Luis Natera Cisneros MD and Juan Sarasquete Reiriz MD. "Arthroscopic-Assisted Management of Unstable Distal-Third Clavicle Fractures: Conoid Ligament Reconstruction and Fracture Cerclage With Sutures". Arthroscopy Techniques 4.6 (2015): e655-e661.

14. Pujol N., et al. "Arthroscopic treatment of comminuted distal clavicle fractures (Latarjet fractures) using 2 double-button devices". Arthroscopy Techniques 2 (2013): e61-e63.

15. Checchia SL., et al. "Treatment of distal clavicle fractures using an arthroscopic technique". Journal of Shoulder and Elbow Surgery 17 (2008): 395-398.

16. Jerosch J., et al. "Which stabilization technique corrects anatomy best in patients with AC-separation? An experimental study". Knee Surgery, Sports Traumatology, Arthroscopy 7 (1999): 365-372.

17. Van der Meijden OA., et al. "Treatment of clavicle fractures: Current concepts review". Journal of Shoulder and Elbow Surgery 21 (2012): 423-429. 
18. Madsen W., et al. "Addition of a suture anchor for coracoclavicular fixation to a superior locking plate improves stability of type IIB distal clavicle fractures". Arthroscopy 29 (2013): 998-1004.

19. Hanflik A., et al. "Open reduction internal fixation of distal clavicle fracture with supplementary button coracoclavicular fixation". Arthroscopy Techniques 3 (2014): 551-554.

20. Levy 0. "Simple, minimally invasive surgical technique for treatment of type 2 fractures of the distal clavicle". Journal of Shoulder and Elbow Surgery 12 (2003): 24-28.

21. Shin S.J., et al. "Treatment of unstable distal clavicle fractures using two suture anchors and suture tension bands". Injury 40 (2009): 1308-1312.

22. Paolo Arrigoni MD., et al. "Associated Lesions Requiring Additional Surgical Treatment in Grade 3 Acromioclavicular Joint Dislocations". Arthroscopy 30 (2014): 6-10.

Volume 2 Issue 9 September 2019 (C) All rights are reserved by Pablo Cañete San Pastor. 